\title{
Para la propagación de la Fe, para el servicio del rey, y utilidad de sus estados y vasallos. Proyecto anónimo para la instalación de una misión en las Islas Malvinas alrededor de 1766.
}

\author{
Para la propagación de la Fe, para el servicio del rey, y utilidad \\ de sus estados y vasallos. Anonymous project for the installation of a \\ mission in the Falkland Islands about 1766.
}

Joaquín García Insausti*

Resumen: En este artículo se analiza brevemente un documento anónimo en el cual se exponen los beneficios que conllevaría la instalación de una misión y un establecimiento naval en las Islas Malvinas. Dos son los ejes sobre los que se centró el análisis de dicho proyecto. El primero aborda el fuerte contenido geopolítico del mismo, coherente con las reformas promovidas por la Corona. Mientras que el segundo indaga sobre el complejo aparato crítico que le sirve de apoyatura, el cual da cuenta la vasta red informativa a la que tenía acceso el autor como miembro de la Compañía de Jesús.

Palabras claves: Islas Malvinas, misiones jesuíticas, Reformismo Borbónico

Abstract: In this article is analyzed briefly an anonymous document in which are exposed the benefits that would carry the installation of a mission and a naval establishment in the Falkland Islands. Two are the topics on which the analysis of the abovementioned project is centered. First will be considered the strong geopolitical content of it, coherent with the reforms promoted by the Crown. Secondly will be taken into account the complex critical apparatus that is used as support, which makes us realize the vast informative network to which the author had access as a member of Jesus' Company.

* Licenciado en Historia por la Universidad Nacional del Sur (Argentina).

E-mail: garciainsausti.j@gmail.com

208 Joaquín García Insausti. Para la propagación de la Fe: Para el servicio del rey ...208-226. 
Key words: Falkland Islands, Jesuitical missions, Bourbon Reforms

Recibido: 5 de agosto de 2016

Evaluado: 18 de diciembre de 2016 


\section{Introducción}

El documento sine data y de autor anónimo que se presentará a continuación se encuentra actualmente depositado en el Archivo Nacional de Chile (Fondo Jesuitas, Volumen 93, 162-168). Sus seis folios escritos en anverso y reverso han sido textualmente transcriptos respetando la grafía original. En él se exponen los beneficios que conllevaría la instalación de un establecimiento naval y una misión en las Islas Malvinas que sirva de base para la evangelización de "la parte mas meridional de la America".

A pesar de la falta de información explícita en el texto del memorial, es posible aproximarse por otra vía a su fecha de redacción. Dos acontecimientos que, por su importancia cumplen un rol fundamental en la argumentación a favor de la fundación propuesta, permiten establecerla de manera bastante precisa: el primero es una referencia al naufragio del navío La Concepción ${ }^{1}$ y el segundo la expedición a Malvinas de Louis Antoine Conde de Bougainville ${ }^{2}$.

Dado que la pérdida de la nave aconteció en enero de 1765, la afirmación de que ese evento ocurrió "el año pasado" contenida en el documento, señala a 1766 como el de su producción. Asimismo, el autor del escrito expresa dudas de que los franceses tuvieran verdadera voluntad de entregar la colonia, hecho que, contrariando esa presunción, se consumaría el uno de abril de 1767, fecha en que Luis XV la puso en manos de la corona española.

La combinación de ambos elementos acrecienta al máximo la probabilidad de que 1766, pocos meses antes de la expulsión de la orden jesuita ${ }^{3}$ de los territorios españoles, sea efectivamente el momento en que se volcó por escrito esta idea de establecer una misión en Malvinas.

\footnotetext{
${ }^{1}$ Se trata del navío de registro Purísima Concepción que zarpó de Montevideo el 6 de diciembre de 1764 con destino al Perú. Llevaba 193 hombres a bordo, entre tripulantes y pasajeros, con caudales que debían ser entregados en el Callao. A principios de enero de 1765 y a raíz de un maniobrar negligente, la nave encalló en la costa norte de la actual Península Mitre (extremo sureste de la Isla Grande de Tierra del Fuego). Los sobrevivientes alcanzaron tierra en una caleta a la que pusieron por nombre Puerto de la Consolación. Auxiliados por los indígenas del lugar (haush), permanecieron allí ochenta días durante los cuales construyeron una pequeña goleta (Nuestra Real Capitana S. Joseph y las Animas), a bordo de la cual retornaron al Rio de la Plata. Un relato de los sucesos se encuentra en: Ratto, 1930. El pecio de la Purísima Concepción fue hallado a principios de 2014 en Caleta Falsa por un grupo investigadores pertenecientes al Programa de Arqueología Subacuática del Instituto Nacional de Antropología y Pensamiento Latinoamericano. Para más información consultar: Elkin, 2014: 39; y http://www.lanacion.com.ar/1669857-hallaron-restos-de-un-barco-espanol-hundido-en-1765

${ }^{2}$ Louis Antoine de Bougainville (1729-1811), militar, explorador y navegante francés famoso por sus contribuciones a la ciencia y la geografía durante la segunda mitad del siglo XVIII. Como se verá a continuación, en 1764 tomó posesión de las Malvinas para la corona de Francia. No obstante, pocos años más tarde las islas fueron vendidas a España y entregadas en 1767 al enviado de Madrid por el propio Bougainville, quien desocupó el establecimiento que había fundado en ellas (Port Louis) y continuó luego la primera expedición francesa de circunnavegación (Caviglia, 2012: 94-101)

${ }^{3}$ Sobre las actividades jesuíticas en América, nos reduciremos a citar las obras introductorias de Ángel Santos Hernández (1992) y Jonathan Wright (2005), y en particular con respecto al Río de la Plata, el clásico trabajo de Magnus Mörner (1986). En lo referente a la evangelización de los indígenas también en Río de la Plata y en el sur de Chile, se destacan las contribuciones del prolífico Guillermo Furlong -entre ellas Furlong, 1943 y 1984-, y otras más recientes como Rolf Föerster (1996) y de Jorge Pinto Rodríguez $(1988 ; 1991)$.
}

210 Joaquín García Insausti. Para la propagación de la Fe: Para el servicio del rey ...208-226. 
El memorial tampoco contiene datos que permitan identificar a su redactor. Sin embargo, el análisis de otros proyectos contemporáneos hace viable una aproximación a su posible contexto de producción. En 1764, el padre Juan Nepomuceno Walter ${ }^{4}$ presentó una iniciativa para restablecer la misión de Nahuel Huapi ${ }^{5}$ y desde allí alcanzar luego el Estrecho de Magallanes, en un plan evangelizador de largo alcance que aspiraba asimismo a habilitar, en el sur de Chile, el paso continental hacia el archipiélago de Chiloé $^{6}$. Entre sus previsiones, que combinaban la tarea evangelizadora con el control soberano de los territorios meridionales, se contaba también el establecimiento de una misión en Tierra del Fuego:

"el establecimiento de una misión en Tierra del Fuego no solo fuera de provecho a sus isleños, sino también de mucha utilidad a la corona y de mucho alivio, consuelo y, algunas veces, de remedio a los navios españoles que pasan frecuentemente por el Estrecho de Maire en cuyas cercanias habria de fundarse, para que los navios que forzosamente se acercan a la costa, al pasar el estrecho, pudieran sin dificultad llegar al puerto y socorrerse mutuamente de las cosas necesarias y para (que) en tiempo de guerra sirviera de mucho freno a los enemigos un fuerte alli mismo, debajo del que pudieran abrigarse nuestros navios y disponerse para salir de refresco contra los enemigos y embarazarles el paso al mar del sur.",

La estrategia ideada comenzó a desarrollarse de manera bastante exitosa con el aval del gobernador de Chile Antonio Guill y Gonzaga ${ }^{8}$, pero la expulsión de la orden en 1767 le puso abrupto término ${ }^{9}$.

A pesar de ciertas similitudes existentes entre el plan del padre Walter y el documento que se presentará a continuación con respecto a los objetivos propuestos, sería excesivo afirmar sin más, que aquel sea el autor de ambos: básicamente porque la idea

\footnotetext{
${ }^{4}$ Juan Nepomuceno, Walther von Walthausen Roth, (1713-1779) llegó como sacerdote a Chile en 1748. Fue padre espiritual de los hermanos coadjutores alemanes en el Colegio Máximo, superior de la residencia de Arauco. Habiendo enfermado pasó al Colegio Máximo como procurador general de la Provincia de Chile hasta la expulsión en 1767 (Hanisch Espíndola, 1972: 324, Storni, 1980: 309).

${ }^{5}$ La misión del Nahuel Huapi funcionó en dos etapas entre fines del siglo XVII y principios del XVIII. La primera transcurrió entre 1670 y 1674, a cargo del padre Nicolás Mascardi. Este encabezó una incursión apostólica con la intención de restituir un grupo de indígenas poyas que se encontraban esclavizados en Chiloé y reconocer el territorio. Mascardi permaneció en la zona adoctrinando y bautizando a sus habitantes hasta su trágica muerte, acontecimiento que precipitó el fin de la primera experiencia misional. La segunda se inició en 1703 con la refundación realizada por el padre Felipe de la Laguna. A pesar de su mayor vigencia en el tiempo, este intento se vio signado por penurias y conflictos. El aumento de las dificultades y la muerte de varios de los misioneros hizo que, tras varias idas y venidas, la misión fuera finalmente abandonada en 1717, luego de ser saqueada e incendiada por los indígenas (Nicoletti, 2014: 44-48).

${ }^{6}$ Page, 2013: 28-29.

${ }^{7}$ Fragmento del "Plan presentado por el P. Juan Nepomuceno Walter a la Junta de Poblaciones el 9 de enero de 1764”, transcripto en Hanisch Espíndola, 1982: 201-209. El resaltado me pertenece.

${ }^{8}$ Antonio de Guill y Gonzaga (1715-1768) ejerció los cargos de gobernador de Panamá (1758-1761) y de Chile (1761-1768). Durante el desempeño de este último, favoreció la fundación de nuevos poblados fronterizos como Rere (1765), Tucapel el Nuevo (1765) y Yumbel (1766) y brindó apoyo a las iniciativas misionales jesuitas, cuya expulsión, sin embargo, debió controlar posteriormente (Medina, 1906: 383384).

${ }^{9}$ Furlong, 1943: 140.
} 
de Walter consistió en establecer una misión evangelizadora y un fuerte en Tierra del Fuego -aduciendo la buena predisposición de los indígenas, de quienes tenía una visión positiva- para auxilio de los navegantes y defensa de la zona en circunstancias de conflicto, mientras que -como se verá- no era exactamente este propósito de nuestro autor anónimo.

Sin embargo, resulta interesante destacar que, a pesar de las diferencias entre ellas, las dos iniciativas revelan un fuerte interés por la geopolítica imperial, demostrando una vez más la influencia que la Compañía de Jesús procuraba ejercer en la administración colonial hasta el momento mismo de su expulsión. Los dos textos exponen, en efecto, una idea que se relaciona estrechamente con las reformas promovidas desde Madrid, en particular el mejoramiento de la defensa de las colonias.

Ante la creciente presencia de naciones extranjeras -sobre todo ingleses y franceses- que intentaban establecerse en la zona del Atlántico sur con el fin de dominar el paso interoceánico, el anónimo redactor del documento que comentamos aspiraba a persuadir al rey de que una colonia en las islas llenaría un triple propósito: primero, instalar una base misional para la evangelización de los indígenas patagónicos y fueguinos; segundo, disponer de un puerto para la reparación y reabastecimiento de los navíos que se dispusieran a realizar la travesía hacia el Pacífico; y por último, estacionar en la región una guarnición militar que resguardase la soberanía española. En ese orden de ideas, argumentaba que proceder del modo que sugiere sería muy beneficioso " $p$. la propagación del Fé I para el Servicio del Rey, y utilidad de sus Estados. Y Vasallos" y tal como veremos a continuación, el desarrollo de cada una de las funciones propuestas revela algunos aspectos interesantes.

En primera instancia y teniendo en cuenta la fuerte impronta misional de la orden, llama la atención que el propósito evangelizador sea tratado de manera tangencial, dando cuenta de un marcado desconocimiento acerca de las características de los indígenas patagónicos. De estos, el autor revela tener sólo vagas noticias gracias a los relatos de los nativos de la región pampeana y del navegante holandés Oliver Van Noort ${ }^{10}$. A pesar de ello y del peligro que, de ser verdadero, hubiera conllevado un supuesto canibalismo de los fueguinos, se insiste en que el establecimiento de la misión en Malvinas sería beneficioso en tanto permitiera que la futura predicación se desarrollarse sin los "vicios" propios de un contacto cercano con pobladores en general y particularmente con hombres de armas.

Respecto a la importancia de la fundación de un puerto seguro, remarca -en vista del reciente naufragio de la Purísima Concepción- la carencia de puntos de recalada en la costa atlántica al sur de Buenos Aires, que obligaba a optar por dos alternativas problemáticas: ingresar en el Rio de la Plata -"Infierno de Navegantes"- o bastante más al norte, en Rio de Janeiro, en este caso con las dificultades propias de una escala en territorio extranjero. Por lo tanto, un establecimiento próximo a las vías de ingreso al océano Pacífico solucionaría los inconvenientes, complementándose a su vez con las posibilidades ofrecidas por Valdivia en el litoral chileno.

\footnotetext{
${ }^{10}$ Oliver Van Noort (1558-1627) fue un navegante y pirata neerlandés, el primero de esta nacionalidad en circunnavegar el mundo. En 1612, van Noort publicó una descripción de ese viaje, en la que incluyó las aludidas referencias a los nativos de la Patagonia continental e insular, con quienes había mantenido contactos violentos y a los que, por este motivo, describe en términos poco benévolos (Caviglia, 2012: 65$67)$.
} 
Finalmente y en lo relativo a la reafirmación de la soberanía hispana sobre la zona, el texto observa que no sólo los franceses estaban interesados en establecerse definitivamente en el archipiélago, sino también que los ingleses habían despachado expediciones con la misma intención. En enero de 1764, el comodoro John Byron ${ }^{11}$, en cumplimiento de las instrucciones que Londres le impartiera, se estableció en Port Egmont, reclamando derechos para su rey ${ }^{12}$. Frente a esta situación de competencia por el control de las islas, la propuesta de establecer un puesto que permitiera conservarlo para Madrid al tiempo que servía de punto de apoyo a la actividad misional encuadraba perfectamente dentro de las novedosas perspectivas propugnadas por Carlos III.

El tratamiento de estas líneas argumentales demuestra el aspecto más importante del documento, esto es, la racionalidad geopolítica subyacente a la "idea de un establecimiento y mission". En este sentido, y tal como ya se comentó, el objetivo misional del proyectado establecimiento, someramente considerado, se subordinaba a la necesidad de fundar un puerto y dotarlo de una guarnición militar. Las prioridades establecidas por el autor en su discurso revelan capacidad de análisis de la realidad política internacional y demuestran que la orden persistía en su intento de influir sobre el poder real de una manera que resultase conveniente para sus objetivos, aún en los momentos previos a su expulsión de los dominios hispanos.

Otro de los elementos que vale la pena destacar es el aparato crítico que apoya el planteo propuesto y que da cuenta del hábil manejo de la vasta red informativa a la que tenían acceso los ignacianos. Se registran un total de veintiuna citas, en las cuales se referencia una amplia variedad de fuentes, clasificables en tres grupos.

El primero está constituido por publicaciones periódicas. El Mercurio de Madrid de agosto de 1764 y la Gazeta de Lugano de septiembre 1765 son mencionados para brindar datos e información fresca referentes a las expediciones de Bougainville y al estado de su colonia.

El segundo es de tipo legislativo. Se echa mano en este caso a la Recopilación de Leyes de los Reynos de Indias (1680) para conferir un marco jurídico y legal al proyecto de misión. Todas las referencias pertenecen al Libro 4, que "Concierne al descubrimiento y la conquista territorial. Fija las normas de población, reparto de tierras, obras públicas y minería" y dentro de él, a los títulos 1, 2 y 4, alusivos a los descubrimientos en general, a los descubrimientos por mar, y a las pacificaciones, respectivamente, con el objetivo principal de establecer y asegurar una clara separación de las órbitas de ac-

${ }^{11}$ El marino inglés John Byron (1723-1786), que llegaría a ocupar altos cargos en la armada británica, navegó por primera vez en aguas del Atlántico meridional en 1740, incorporado a la expedición encabezada por el comodoro George Anson y enviada para hostilizar las posesiones españolas de la costa pacífica de América del Sur (Villar, 2004: 15-17). Byron era por entonces un joven guardiamarina embarcado en la fragata HMS Wager. Esta nave naufragó en mayo de 1741 en el Archipiélago de las Guaytecas, frente a la costa pacífica austral de Chile (mayor información sobre la expedición Anson, la pérdida de la Wager y las desventuras de los sobrevivientes en Walter, 1748; Morris 2004 [1751] y Villar, 2004, entre otros; también se encuentra disponible el relato del mismo Byron -1996 [1768]-acerca de las peripecias sufridas en esa oportunidad. A lo largo de los años 1764 a 1766, al comando del HMS Dolphin, Byron realizó un viaje de circunnavegación durante el cual desembarcó en las Islas Malvinas casi al tiempo que se concretaba la entrega del archipiélago a los españoles por parte de Bougainville (Caviglia, 2012: 105107).

${ }^{12}$ Couyoumdjian, 1971: 62.

213 Joaquín García Insausti. Para la propagación de la Fe: Para el servicio del rey ...208-226. 
tuación, de modo que la labor de los misioneros no se viera interferida por el personal militar que los acompañe.

Por último, el tercer grupo está conformado por una serie de obras editas historias, cronologías y diccionarios- que ofrecen información general acerca del medio natural, los nativos del área y las dificultades que presentaba la travesía interoceánica.

Entre los libros mencionados -algunos de ellos en más de una ocasión- sobresalen dos obras del jesuita francés Pierre François Xavier de Charlevoix ${ }^{13}$, tituladas Fastes chronologiques de la découverte du Nouveau Monde" (se cita como Fastes du N. Monde) e "Histoire du Paraguay" (en la transcripción como Historia dei Parag.), solo disponibles en francés al momento de redacción del documento. La primera de ellas consiste en un resumen cronológico de los acontecimientos más importantes relativos a los descubrimientos realizados a partir del siglo XIV, y se encuentra incorporada al primer tomo de la segunda edición de "Histoire et description générale du Japon" publicada en 1736. Por su parte, la "Histoire du Paraguay" fue editada en ocho tomos el año 1757, de los cuales se citan partes del I y el VI.

Las dos restantes hacen referencia a Tierra del Fuego y el autor anónimo accedió a sus traducciones al italiano. Una de ellas es "Dizionario geográfico portatile" (abreviado como Diccionar. Geograph.) también de 1757, versión del Dictionnaire Géographique Portatif (1749), del que se cita la entrada "Foco" (fuego) que trata sobre aquella gran isla y sus habitantes, a quienes se les atribuyen inexistentes prácticas antropofágicas. La otra es "Storia degli Stabilimenti Europei in America" (Storia degli Stabili Europ. in Amer. en la transcripción) traslación al italiano de la obra titulada An account of the European settlements in America" (1763) atribuida a Edmund Burke ${ }^{14} \mathrm{y}$ publicada originalmente en $1757^{15}$, de la cual el autor de nuestro documento extrajo información que le permitió subrayar la peligrosidad de la navegación interoceánica y la inutilidad del establecimiento de la misión en aquella gran isla.

El conocimiento y utilización de este conjunto de materiales publicados relativamente poco tiempo antes de la elaboración de la "Idea del establecimiento y misión", además de reforzar nuestra afirmación previa acerca del caudal informativo al que tenían acceso los miembros de la orden, revela el interés principal del redactor que apuntaba a fines más terrenales que la evangelización de los nativos americanos. Así puede definirse este intento de propiciar transformaciones en la geopolítica española, dentro de un contexto en el que Francia e Inglaterra, sobre todo esta última, habían comenzado a definir su perfil de potencias internacionales.

La expulsión de los jesuitas, contemporánea con la evacuación de Port Louis y la entrega de Malvinas por parte de los franceses -hechos casualmente ocurridos el 1 y 2

\footnotetext{
${ }^{13}$ Charlevoix (1682-1761) fue un reconocido jesuita, viajero e historiador. Entre 1720 y 1722 encabezó una misión encargada de explorar las posesiones francesas en América del Norte y delimitar algunas de ellas. La posterior difusión de su Histoire et description générale de la Nouvelle France implicó que se lo reconociera como el primer historiador de la Nueva Francia. Publicó además numerosas obras de carácter histórico, entre las que se destacan aquellas citadas en la transcripción que analizamos.

${ }^{14}$ Edmund Burke (1729 - 1797) fue un escritor, filósofo y político irlandés, mentor del liberalismo moderado y muy crítico de los fundamentos ideológicos y las prácticas de la Revolución francesa, objeciones que expuso en su obra más conocida, titulada Reflections on the Revolution in France (1790).

${ }^{15}$ La edición más antigua a la que pudimos acceder es: Burke, 1760.
} 
de abril de 1767 respectivamente- implicó que el proyecto colonizador propuesto no se realizara. La corona española mantuvo en general una actitud de desinterés y de relativa desprotección sobre la Patagonia y sus dominios atlánticos australes.

No obstante, pocos años más tarde, una lectura geopolítica de las propuestas de otro jesuita, el inglés Thomas Falkner ${ }^{16}$, tendría el efecto de promover algunos cambios en esa actitud. En 1774, Falkner publicó su libro titulado A Description of Patagonia and the adjoining parts of South America, with a grammar and a short vocabulary, and some particulars relating to Falkland's Islands ${ }^{17}$ en el que asumía que el curso del río Negro podría ser remontado con relativa facilidad hasta llegar a Valdivia y destacaba los beneficios que reportaría el establecimiento de una colonia en su desembocadura. En respuesta a lo que se interpretó desde Madrid como una expresión de las intenciones expansivas de los británicos, se puso en marcha una política de colonización que, aunque no fue todo lo exitosa que se esperaba, creó un resguardo en la boca del río con la fundación del Fuerte del Carmen en $1776^{18}$.

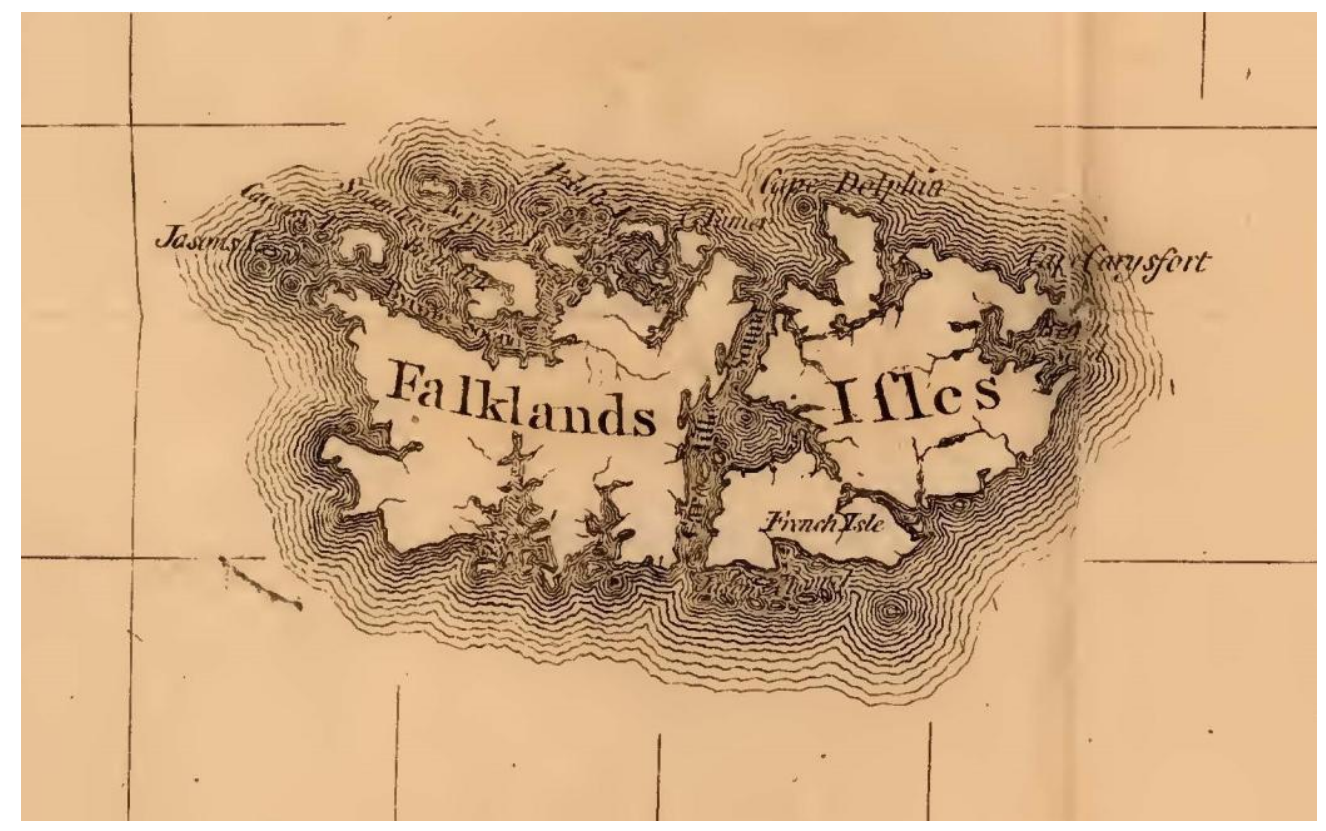

Fragmento del mapa de la Patagonia que acompaña la primera edición inglesa del texto del P. Falkner en donde se ven las Islas Malvinas

\footnotetext{
${ }^{16}$ Thomas Falkner (1702-1784) llegó a Buenos Aires en 1730 como médico de un barco dedicado a la trata esclavista. Mientras permanecía en la ciudad cayó gravemente enfermo y luego de su recuperación, renunció a su pertenencia calvinista para adherir a la fe católica e ingresó a la Compañía de Jesús ordenándose sacerdote en 1739. Entre 1740 y 1744 residió en Santiago del Estero y Tucumán, encargándose más tarde de los efímeros intentos de evangelización de los indígenas del territorio pampeano y nordpatagónico, actividad que desempeñó hasta 1751. A partir de 1756 y hasta el momento de la expulsión, se dedicó a la enseñanza de matemática en la Universidad de Córdoba. Regresó finalmente a Inglaterra en 1771, y allí prestó servicios de capellán en varias casas de la nobleza. Su citada obra de 1774 resulta de ineludible consulta para quienes se interesen en el conocimiento de las sociedades nativas de la región (Mandrini, 2003: 20-27).

${ }^{17}$ Falkner, 1774.

${ }^{18}$ Couyoumdjian, 1971: 71-73.
}

215 Joaquín García Insausti. Para la propagación de la Fe: Para el servicio del rey ...208-226. 


\section{Transcripción de "Fondo jesuitas, Volumen 93, 162-168"}

\section{[162]}

\section{Idea de un Establecimiento y Mission en la parte mas meridional de la America}

Entre los grados 305. y 325. de longitud 36 y 54 de latitud meridional tiene el Rey dominios dos veces al menos mas dilatados $q^{\text {e. }}$ toda España: y habiendo en ellos tanto $\mathrm{q}^{\text {e. }}$ guardar, tanto $\mathrm{q}^{\mathrm{e}}$ defender, tanto $\mathrm{q}^{\text {e. }}$ disfrutar, $\mathrm{y}$ tantas naciones infieles $\mathrm{q}^{\text {e. }}$ traer al conocim ${ }^{\text {to. }}$ de Dios, es mui visible la falta $\mathrm{q}^{\text {e. }}$ hace algún Establecim ${ }^{\text {to. }}$, y Mision, pues en todo aquel Espacio no hay un passo ocupado de Españoles, aunque todo sin disputa es parte de su dominación. Y uno o dos Establecim ${ }^{\text {tos. }} q^{\text {e. }}$ hubo en tiempo, y en lugar $q^{\text {e. }}$ eran menos necesarios, se abandonaron; y dexaron de ser.

En las tierras q $\mathrm{q}^{\mathrm{e}}$ desde las ultimas poblaciones de la Governacion del Rio de la Plata se estienden hasta el Estrecho de Magallanes en la tierra q $q^{\text {e. }}$ llaman del Fuego, y en las nuevas islas, $\mathrm{q}^{\mathrm{e}}$ unos llaman de la Asumpcion, otros de Anican, otros Maloinas, o Malvinas, hai noticias modernas qe havita numero crecido de Infieles pacificos, cuio genio sencillo, y ageno de las Impressiones $q^{\text {e. }}$ ocasiona el trato, da Esperanza de convertirse a la fé. Estas mismas Islas, y tierras caen en la mediación del Camino, $\mathrm{q}^{\mathrm{e} .}$ hacen los Navios de España a los Puertos en el Mar del Sur, y del q $\mathrm{q}^{\mathrm{e}}$ pudieran hacer para ir a las otras indias, y a Philippinas por el Occidente, o también $\mathrm{p}^{\mathrm{r} .}$ el cabo de BuenaEsperanza. Son tierras en cuias costas las Naciones Estrangeras han intentado Estable-

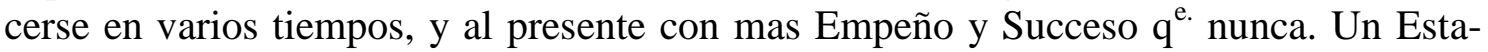
blecimiento, y una Mission ordenada a la Conversion, y pacificación de los Infieles dichos. Un Puerto, q e. fuese escala en la navegación al Mar del Sur, y de la India y Recurso en casso necesario a los Navios Españoles, $q^{\text {e. }}$ la practicasen. Un Establecim ${ }^{\text {to. }}, q^{\text {e. }}$ impidiese los Establecimientos Estrangeros en tan importantes, y dilatadas costas, y tierras abandonadas, seria de la maior consecuencia $\mathrm{p}^{\mathrm{a}}$ la propagación del Fé

[162 vta.] para el Servicio del Rey, y utilidad de sus Estados. Y Vasallos.

El año de 1745 ordeno S. M., q. se Reconociese la Costa de los Patagones, o de la tierra Magallanica con intento de $\mathrm{q}^{\mathrm{e} .}$ se plantease semejante Establecim ${ }^{\text {to. }} \mathrm{p}^{\mathrm{a} .}$ los Espresados fines. Reconociendose hasta el Estrecho, sin pasar a la tierra del Fuego, y sin haber noticia de las Nuevas Islas, $\mathrm{q}^{\mathrm{e}}$ en distancia de no muchas leguas caen al oriente. El Reconocimiento hasta el Estrecho se hizo en la maior parte por agua, y los q ${ }^{\text {e. }}$ saltaron en tierra, no se apartaron dela Costa, y como se halló desierta, sin aguadas permanentes, y cubierta de Medanos, y Arenales, se calificó de Inutil, e intransitable. Falto Sitio Oportuno, por $\mathrm{q}^{\mathrm{e} .}$ falto la perseverancia en buscarlo.

Tampoco se hallaron habitantes: con $\mathrm{q}^{\mathrm{e}}$ los dos Missioneros, $\mathrm{q}^{\mathrm{e}}$ iban en la Embarcacion destinados $\mathrm{p}^{\mathrm{a} .}$ quedarse trabajando en su Conversion, se volbieron tanvien Inutilmente con los descubridores al mismo lugar de donde, y como habían salido. Habitantes hai sin duda; pero están Retirados de la Costa en las tierras, q e. se Riegan, y fecundizan con las Vertientes de la Cordillera, y en sus Valles, de donde en las Estaciones del año en $\mathrm{q}^{\mathrm{e}}$ saben $\mathrm{q}^{\mathrm{e}}$. no han de faltar aguadas, y caza, salen, y se acercan a las Costas del Mar del Norte. Aquí los vió, y se batió con ellos la gente de la Armada del Holandes Oliverio Van Noort el año 1599. \{1\} Y en nuestros días la gente de Buenos Aires, $\mathrm{q}^{\mathrm{e}}$ en la Lancha de Dn. Antonio Arriaga fue por Sal a la Bahia de S. Julian el año de 1758, los vio, y los trato, dando los barbaros el tiempo qe. allí se detubo, especiales 
muestras de natural pacifico, y docil. Y los Indios Aucáes, y los Pampas fronterizos del Rio de la Plata y de Mendoza contestes afirman, $\mathrm{q}^{\mathrm{e}}$ hacia el Sur son sin numero los Toelchus, los Poyas, y mas los Indios de apie. Todos los quales, por no haver sentido los agravios verdaderos, o falsos, $\mathrm{q}^{\mathrm{e} .}$ pretenden los fronterizos haver Recivido de los Españoles están mas dispuestos, para oir Razon, y las verdades de la fé.

Los q el el año pasado de 1765 naufragaron en el Navio

\{1\} Memor. de Frev. Año de 1705. Art CXLVII

[163] la Concepcion, haciendo viage al Mar del Sur, en la Costa de la tierra del Fuego bieron, y trataron Indios de genio igualmente dócil, y pacifico, $\mathrm{q}^{\mathrm{e} .}$ les dieron socorro en su desgracia: fuera de los mismos, $\mathrm{q}^{\mathrm{e}}$ en varios tiempos se han alcanzado a ver en aquella tierra, y q $\mathrm{e}^{\mathrm{e}}$ le hicieron dar el nombre de tierra del Fuego, y muestran que no esta deshabitada.

De las Nuevas Yslas llamadas Maloinas, o Malvinas refieren las noticias publicadas del año pasado de 1765, que los isleños habían hecho alianza con los Franceses $\{2\}, \mathrm{q}^{\text {e. }}$ se aprovecharon de nuestra omission Estableciendose allí. Y si estas Islas, o tierras son tan grandes, y de un Clima tan sano, y benigno, tan abundantes de todo lo necesario $\mathrm{p}^{\mathrm{a} .}$ la vida, como se ha publicado, puede discurrirse, $\mathrm{q}^{\mathrm{e}}$ no son pocos los Naturales, $\mathrm{q}^{\mathrm{e} .}$ las havitan

El Capitan Peré de S. Maló ${ }^{19}$ las costeo p pr. 50 leguas $\{3\}$ El Cavallero Ricardo Hawkins ${ }^{20}$ las costeó por $60\{4\}$ Mr de Bougainville contó 200 leguas de país \{5\}, aunque no se ha Reconocido todas. Y halló en su Segundo viage, $q^{\text {e. }}$ de 115 nuebos pobladores, $\mathrm{q}^{\mathrm{e}}$ en el primero havia conducido, ninguno havia Enfermado $\{6\}$. Si para los Estrangeros Recien llegados es tan benigno el Clima, que será para los Naturales?

Aunque en la tierra Magallanica, en la del Fuego, en las nuevas Islas no hubiera Infieles $q^{\text {e. }}$ traer al conocimiento de Dios, solo por impedir, $\mathrm{q}^{\mathrm{e}}$ otras naciones, o Enemigos de España o q $\mathrm{e}^{\mathrm{e}}$ algún tiempo lo pueden ser, se Estableciesen en ellas, o Establecidas se arraigasen multiplicando Establecim ${ }^{\text {tos. }}$; seria conveniente y necesario, $q^{\text {e. España no }}$ las dejase abandonadas. Las naciones Estrangeras han llegado a gustar, y palpar las utilidades de un Establecim ${ }^{\text {to. }}$ En aquellas partes, asi $\mathrm{p}^{\mathrm{r} .}$ las producciones de $\mathrm{q}^{\text {e. }}$ el terreno es capaz, y fecundo, como $\mathrm{p}^{\mathrm{r} .}$ las comodidades del Comercio. Haviendo tomado gusto, no desistirán mientras las vean desocupadas

\{2\} Gazet. de lugano de 2 de sept. 1765. Parigi.

\{3\} P. Charlevoix. Fastes du N. Monde Amn 1700

\{4\} Charlev. Ibid

\{5\} Mercur. de Madrid, de Agosto 1764. París

\footnotetext{
${ }^{19}$ Pierre Perrée du Coudray de la Villestreux (1656-1742), navegante y corsario francés, originario de Saint-Malo. En 1703 partió de dicha ciudad al comando del Saint-Charles rumbo al Callao, travesía durante la cual, al regresar por el Cabo de Hornos, se aproximó a las Malvinas, reconociendo una isla grande flanqueada al este por otras más pequeñas (Caviglia, 2012: 75).

${ }^{20}$ Famoso por sus acciones contra las posesiones españolas en Sudamérica, el navegante y corsario inglés Richard Hawkins (1562-1622) afirmó haber descubierto a principios de febrero de 1594 un grupo de islas a las que bautizó Hawkins' Maiden Land y que posiblemente fueran las Malvinas (Caviglia, 2012: 56-57).
} 
$\{6\}$ Gazet. de Lugano. Cit

[163 vta.] Podrá el Rey alegar títulos de propiedad; pero es cosa sabida q q $^{\text {e. }}$ los Estrangeros en quanto a los dominios de la America, no quieren Reconocer otro titulo q el $\mathrm{q}^{\mathrm{e}}$. nace de la ocupación; y posession. Y aunque el Embajador de S. M. en Paris, según es forma, haia Reclamado contra el Establecim ${ }^{\text {to. }}$ de $\mathrm{Mr}$ de Bougainville mencionado; es natural $\mathrm{q}^{\text {e. }}$ disimulando se trabaje en Cimentarlo, y darle cada dia maior firmeza, la $\mathrm{q}^{\text {e. }}$ sin maior Empeño crecería con el tiempo: y al cabo, disimulando España, prescribirá de hecho.

En tiempo de Guerra es mas necesario un Establecim ${ }^{\text {to. }}$ en aquella $p^{\text {te. }}$ porq $^{\text {e. }}$ velando con alguna fuerza, puede estorbar $\mathrm{q}^{\mathrm{e}}$ pase maior armamento a hostilizar las costas del Mar del Sur. Un Moderno Escritor Ingles $\{7\}$ atribuie la seguridad de aquellas costas a lo difícil, y peligroso $\mathrm{q}^{\mathrm{e}}$ es el paso para todo armamento Europeo considerable, $\mathrm{p}^{\mathrm{r}}$. el Estrecho de Magallanes, y por el Cabo de Horno. La loro siccureza (dice) devesi........ al difficile e pericoloso passaggio per qualunque Armamento Europeo, che fosse considerabile fra gli strati Magellanico, e di capo Horn, anzi che alla loro forza, 0 vigilanza $^{21}$. Que fuera si a lo difícil, y arriesgado del paso se juntara alguna fuerza y vigilanzia en aquella parte, por donde forzosamente se ha de pasar? tal es el Estrecho de Maire, o el de Magallanes, (aunque este por su largura, y naturaleza esta bien defendido), enfrente de los quales caen las Nuebas Islas.

Pero es manifiesta la Importancia de un Establecim ${ }^{\text {to. }}$ solido en las inmediaciones de los dos Estrechos, aunque las Naciones Estrangeras abandonasen aquellas costas, y todas las ulteriores, y aunq ${ }^{\text {e. }}$ se supiera de cierto, $q^{\text {e. }}$ en tiempo de guerra no las habían de instalar, ni en tiempo de paz se habían de aprovechar de ellas en prejuicio de España. Desde Cadiz, o sea desde las Canarias hasta los Puertos del Mar del Sur tienen un Mar inmenso $\mathrm{q}^{\mathrm{e}}$ navegar los Navios $\mathrm{q}^{\mathrm{e}}$ van por aquella Carrera. Succedera muchas veces lo $\mathrm{q}^{\mathrm{e}}$. muchas veces ha sucedido, y lo $\mathrm{q}^{\mathrm{e}}$ acaba de suceder con la perdida del Navio la Concepcion, $\mathrm{q}^{\mathrm{e}}$ a la hida, o a la vuelta se hallen los Navegantes necesitados, o de invernar, o de Repararse, o de bastimentos, o de alguna otra cosa de las q $\mathrm{q}^{\mathrm{e}}$ suelen necesitarse

\{7\} Storia degli Stabil. Europ. in Amer. trad in ital. P. 2. Cap. 13.

[164] en tan largas Navegaciones. Quanto importaría tener a la mitad del Camino un Puerto seguro donde recobrarse? Los Ingleses tienen diferentes Escalas para ir a Levante $\mathrm{p}^{\mathrm{r} .}$ el Mediterraneo, y $\mathrm{p}^{\mathrm{r} .}$ los tratados de Comercio les es fácil el arribar a varios Puertos quando navegan a sus Colonias Asiaticas, Tienen también en medio del Mar Atlantico Establecimto. para el mismo fin en la isla de $\mathrm{S}^{\mathrm{ta}}$. Elena. Los Franceses para ir a su Pondicheri $^{22}$ en Oriente, no sabemos $\mathrm{q}^{\text {e. }}$ tengan Establecim ${ }^{\text {to. }}$ desde $\mathrm{q}^{\mathrm{e}}$ se apartan de la Costa Occidental del Africa, habiendo perdido el $\mathrm{q}^{\mathrm{e}}$ tenian en Madagascar; mas por eso mismo q ${ }^{\text {e. }}$ no lo tienen la procuran tener: y este es el fin por $q^{\text {e. }}$ dan calor al establecim ${ }^{\text {to. }}$ $\mathrm{q}^{\mathrm{e} .}$ han hecho en las Nuebas Islas enfrente de la Costa de Patagones. A lo menos este fin les atribuyen las nuevas publicas. Potreverse (dicen) cavar profito dalle sue produccioni;

\footnotetext{
${ }^{21}$ Su seguridad se debe más al difícil y peligroso paso para cualquier tipo de Armamento Europeo considerable por el estrecho de Magallanes y por el Cabo de Hornos, que a su fuerza o vigilancia. Esta traducción del italiano y la siguiente [nota 23] fueron realizadas por la Licenciada Lucía Lasry (Universidad Nacional del Sur) a quien mucho se agradece su colaboración.

${ }^{22}$ Del tamil Pānticcērri (Ciudad Nueva), transpuesto al inglés Pondicherry y al francés Pondichéry, era la antigua denominación del dominio de Francia en Tamil Nadu, sudeste del territorio de la India.
} 
ma si risguarda come un grande vantaggio il potersi aprodare i vascelli, che intrapren$\underline{\text { dono il passaggio alle grandi Indi }}^{23} \cdot\{8\}$ Los Portugueses para ir a su India tienen a Mozambique para Recobrarse. Los Holandeses el Cabo de Buena Esperanza, o el fuerte Cercano, en q ${ }^{\text {e. }}$ se han Establecido. Solo los Españoles no tienen donde Repararse por esta Carrera dilatada del Cabo de Horno, y si quisieren ir asus Philippinas enderechura, y hacer Escala, es necesario $q^{\text {e. }}$ pidan puerto, y en el estén a merced agena.

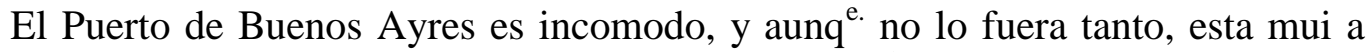
tras mano, y distante de aquellos Rumbos; es dificultosam ${ }^{\text {te. }}$ accesible, y mas para navios averiados, y maltratados con algún contratiempo de los qe. pueden hacer necesario el Recurso, y socorro prompto. Al Rio de la Plata llaman con razón Infierno de Navegantes $\{9\}$ : y es fuerte cosa, $\mathrm{q}^{\text {e. }}$ los navegantes necesitados de Remedio en una larga navegación se vean obligados a buscarlo en el infierno. Con que solo resta a los q. Naufragan en aquella Carrera, o necesitan Recobro buscan

$\{8\}$ Gazet. de Lugano de 2 de Sept. 1765. Parigi

\{9\} P Charlev. Historia dei Parag. lib. 1. Pag 26

[164 vta.] en el Rio Janeyro, o en otro tal, distante también del Rumbo, y Sugeto a otro dueño: Circunstancia, $\mathrm{q}^{\mathrm{e}}$ suele ocasionar atrasos maiores desazones, y quejas, de $\mathrm{q}^{\mathrm{e}}$ por ventura havran llegado algunas a la Corte.

El Establecim ${ }^{\text {to. }}$ ideado no conviene ponerlo en la Costa de Patagones, o de la tierra Magallanica; por $q^{\text {e. }}$, aunq $q^{\text {e. }}$ tierra dentro en los parages, $q^{\text {e. }}$ se Riegan con las vertientes de la Cordillera, se cree $\mathrm{q}^{\mathrm{e}}$ la $\mathrm{q}^{\mathrm{e}}$ ha de ser cabecera principal este a la lengua del agua, para manejarse $\mathrm{p}^{\mathrm{r} .}$ mar, y se logren todos los fines Espresados: y a la lengua del agua en aquella costa no parece haver Sitio apropósito. Los Holandeses en el tiempo q ${ }^{\mathrm{e}}$ infestaban aquellas Costas, y se apoderaron del Brasil, lo buscaron, y no lo hallaron. Y el mismo desengaño parece $\mathrm{q}^{\text {e. }}$ Resulto del Reconocim ${ }^{\text {to. }} \mathrm{q}^{\text {e. }}$ por orden de S. M. se hizo

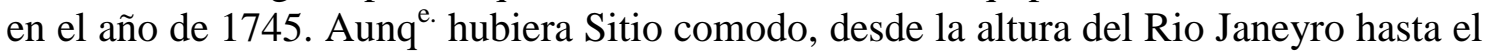
Estrecho se va Retirando la Costa mui al Poniente, para dar despues buelta al Oriente, y acercarse con la tierra del Fuego al passo $q^{\text {e. }}$ se ha de hacer $p^{\text {r. }}$ el Estrecho de Maire: $y$ quanto mas se retira de este modo la Costa, tanto mas se aleja del Rumbo q ${ }^{\text {e. }}$ lleban los Navios del Mar del Sur, y del q $\mathrm{q}^{\mathrm{e}}$ pueden llevar a Philippinas. La tierra adentro de esta Costa, puesto q $\mathrm{q}^{\mathrm{e}}$ la habitan indios pacificos conviene dejarla para el Establecimiento de Misiones $\mathrm{p}^{\mathrm{r} .}$ ahora, $\mathrm{p}^{\mathrm{a} .} \mathrm{q}^{\mathrm{e} .}$ los Misioneros entren solos, y sin Estrepito de armas a anunciarles el Evangelio, fomentando su Ministerio desde el lugar donde se pusiere la Cabezera gral.

Tampoco conviene ponerle en la tierra del Fuego $p^{\text {r. }}$ la misma Razon, y $p^{\text {r. }} q^{\text {e. }}$ escondida entre los dos estrechos de Maire, y de Magallanes en el fondo de la Rinconada, q. forma la punta de la Costa de Patagones, nadie la busca, sino para dejarla, ni es Camino para alguna part ${ }^{\mathrm{e}}$ Cerca de ella se fundo antiguamente sobre el borde Septentrional del Estrecho de Magallanes la Ciudad de S. Phelipe ${ }^{24}$, para velar sobre el passo del

\footnotetext{
${ }^{23}$ Se podría sacar provecho de sus producciones; pero se considera una gran ventaja el hecho de poder atracar las embarcaciones, que emprenden el viaje hacia las grandes Indias [esto es, las Indias Orientales].

${ }^{24}$ San Felipe fue fundado por Pedro Sarmiento de Gamboa el 25 de marzo de 1584 cerca del actual emplazamiento de la ciudad de Punta Arenas. Poblaron el lugar unas trescientas personas que, al poco tiempo, quedaron abandonadas en esa costa inhóspita, a raíz de una sublevación, cuyos protagonistas se apo219 Joaquín García Insausti. Para la propagación de la Fe: Para el servicio del rey ...208-226.
} 
Mar del Sur, pero desde el año 1616 en $\mathrm{q}^{\text {e. }}$ se descubrió passo mejor $\mathrm{p}^{\mathrm{r} .}$ el Estrecho de Maire, nadie quiere pasar por el de Magallanes, y se tubo $\mathrm{p}^{\mathrm{r}}{ }^{\text {in }}$ intil el Empeño de mantener

[165] el Establecimiento de S. Phelipe. Pero la tierra dicha podrá quedar para teatro de Missiones en la manera qe se dijo dela tierra Magallanica.

El Sitio mas conveniente para los tres fines Esperados, es asaber, para la Conversion de los infieles, para la comunicación de las provincias mas Remotas de la dominancion de España, y Reparo de las invasiones q en tiempo de paz, o guerra pudieran intentar las naciones Estrangeras, parece ser en las islas nuevas llamadas Malvinas, supuesto $\mathrm{q}^{\mathrm{e} .}$ los Franceses desistirán de su nuevo Establecim ${ }^{\text {to. }}$ en ellas, como lo han prometido según se dice. $Y$ aunq ${ }^{\text {e. }}$ no desistieran, la amplitud de lo $q^{\text {e. }}$ se ha descubierto según las Relaciones es tanta, $\mathrm{q}^{\mathrm{e}}$ hai cavida $\mathrm{p}^{\mathrm{a} .}$ las dos Naciones. Ambas tienen Colonia en la isla de $S^{\text {to. }}$ Domingo, $\mathrm{q}^{\text {e. }}$ no es tan grande como lo $\mathrm{q}^{\mathrm{e}}$ se ha descubierto delas Mal-

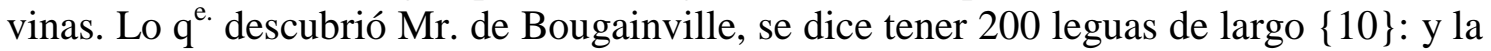
isla de $S^{\text {to. }}$ Domingo en su maior longitud apenas llegaría a 160. Ella dista 80 millas del Continente, o dela Costa de Patagones, q ${ }^{\text {e. }}$ serán como 23 leguas Españolas: distancia moderada, quanto basta para evitar los inconvenientes, $\mathrm{q}^{\text {e. }}$ suele causar el trato de los Españoles con la gente nueva, $\mathrm{q}^{\mathrm{e}}$ se quiere pacificar, y convertir, y $\mathrm{q}^{\mathrm{e}}$ en los principios suele Recelarse, y tal vez Viciarse con la Comunicación mas inmediata. No era necesa-

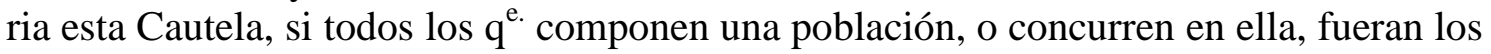
$q^{\text {e. }}$ deben, pero quien inficione, Especialmente a gente nueva, y Sencilla, Y la distancia

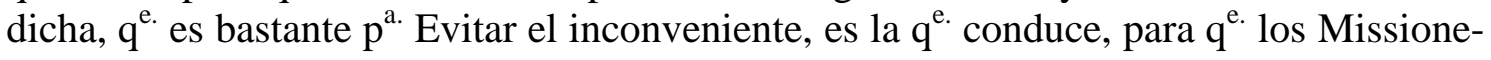
ros, y las Missiones tengan cerca quien les de la mano, y el fomento necesario.

Caen las dichas Yslas enfrente del Estrecho de Magallanes $\{11\}$ : consiguientemente en menor distancia dela tierra del Fuego $q^{\text {e. }}$ de la Costa Magallanica, $p^{\text {r. }}$ lo $q^{\text {e. }}$ se inclina mas hacia el Oriente la Costa $\mathrm{q}^{\mathrm{e}}$ cae al Sur del Estrecho, a donde con

\{10\} Mercur. de Madrid de Agosto 1764: $\underline{\text { Paris }}$.

$\{11\}$ Mercur. de Madrid. Citad.

[165 vta.] mas facilidad se podrá pasar ala pacificacion delos infieles q $\mathrm{q}^{\mathrm{e}}$ la habitan, y a disfrutar las producciones del pais, cuio descubrimiento promete nuevas utilidades al Estado. Por las memorias de los Holandeses $\{12\}, \mathrm{q}^{\mathrm{e}}$ han navegado por aquellas partes parece $\mathrm{q}^{\mathrm{e}}$ lo $\mathrm{q}^{\mathrm{e}}$ llamamos tierra del Fuego es un monton de islas, entre las quales hai paso para navios. $\mathrm{Y}$ pudiera, $\mathrm{q}^{\mathrm{e}}$ se Encontrase algún paso mas comodo, $\mathrm{q}^{\mathrm{e}}$ el paso común por el Estrecho de Maire. Otro escritor dice: la tierra del Fuego es el nombre, $\mathrm{q}^{\mathrm{e}}$ se da a muchas islas, $q^{\text {e. }}$ se Estienden cerca de 130 leguas a lo largo del Estrecho de Magallanes en la America meridional. Hay en ella muchos Valles y praderas Regadas de bellos arroyos habitados de Salvages, delos quales algunos son Comedores de Carne humana. $\{13\}$ En esta calidad de antropófagos, q. se atribuie a los naturales de la tierra del Fuego, aunque sea verdad, no hai cosa, qe. deba arredrar el Celo de los Ministros Evangelicos: y por las Otras, $\mathrm{q}^{\text {e. }}$ se atribuien al terreno, se conoce $\mathrm{q}^{\mathrm{e}}$ no hai en el menos

deraron de tres navíos y partieron dejando a los demás librados a su suerte. Con sólo dos excepciones, los pobladores murieron por inanición. En 1587, el corsario inglés Thomas Cavendish fondeó en la zona, rescató a uno de los sobrevivientes y encontró los restos de los restantes. La trágica experiencia relatada por ese afortunado quedó expresada en la denominación Puerto Hambre (Port Famine) que Cavendish le asignó al lugar.

220 Joaquín García Insausti. Para la propagación de la Fe: Para el servicio del rey ...208-226. 
Razones $\mathrm{p}^{\mathrm{a} .}$ calificar de intransitable la Costa de Patagones. Pero bien esta que por ahora no se trate de poner el Establecim ${ }^{\text {to. }}$ principal en la tierra del Fuego, hasta $\mathrm{q}^{\mathrm{e}}$ poniéndolo en las Malvinas, se descubran con el tiempo maiores conveniencias.

Estas islas, $\mathrm{q}^{\mathrm{e} .}$ por la parte Occidental, por donde se acercan al Estrecho de Magallanes, aun no se han descubierto, caen por los grados 50: 51 y 52 de latitud meridional, como 50 leguas del Estrecho de Maire al Nord-Nord-Est, $\mathrm{q}^{\mathrm{e}}$ es decir, atravesadas en el Rumbo, $\mathrm{q}^{\text {e. }}$ han de tomar los Navios, $\mathrm{q}^{\text {e. }}$ doblando el Cabo de Hornos Salen de aquel Estrecho para venir a España, o los q e. de España salen para la Mar del Sur, Situacion la mas oportuna para los Navios, $\mathrm{q}^{\mathrm{e}}$ van, o buelben necesitados de Reparo, y para guardar aquel único importante paso al Mar del Sur.

\{12\} Charlev. Fastes du N. Monde Amn. 1520

$\{13\}$ Diccionar. Geograph. trad en ital. Edic. Venet. V. Foco

[166] En tanta Concurrencia de Comodidades par la Navegacion, y Comercio de España, para la Seguridad de sus, dominios, para la Conversion delos Americanos, $\mathrm{q}^{\mathrm{e}}$. siempre se ha tenido delante de los ojos, pudiera hacerlas Sospechosas el ser tan Obvias y el no haverse aprovechado de ellas hasta ahora. Como es posible, $\mathrm{q}^{\mathrm{e}}$. en tantos años de navegación por aquella altura, estando casi a la boca del Estrecho por donde todos pasan, ninguno las havia visto; o si las han visto, como es posible, $\mathrm{q}^{\text {e. }}$ ninguna Potencia las haia querido desfrutar? Especialmente en tiempos q ${ }^{\text {e. }}$ tanto Empeño se ha puesto en Registrar, aprovecharse de las tierras mas cercanas a uno, y otro polo, y por eso mas intransitables, y mas inútiles. Esta Consideracion ya no tiene fuerza con lo q esta Sucediendo; pues los Franceses han visto, y observado las nuevas islas, han formado Establecim $^{\text {to. }}$ en ellas, y las están desfrutando, aprovechandose de sus Puertos, de sus Maderas, de sus producciones, de su Situacion, y del trato con los isleños. Asi lo Refieren las nuevas publicas $\{14\}$.

Aquí (dicen, hablando de París) se halla el Cavallero Bougainville de vuelta por la Segunda vez de la isla, $\mathrm{q}^{\mathrm{e}}$ se cree sea parte de las llamadas Malvinas cerca de la tierra de los Patagones, donde havia formado un Establecim ${ }^{\text {to. }}$, $\mathrm{q}^{\text {e. }}$ hallo en el mejor estado. Para dar principio havia dejado 115 pobladores, los quales no han padecido Enfermedad alguna, bien $\mathrm{q}^{\mathrm{e} .}$ su propagación procede con lentitud, $\mathrm{p}^{\mathrm{r} .}$ no haver mas de 15 Mugeres. Dicese, $\mathrm{q}^{\mathrm{e}}$ se ha presentado una tropa de Salvages con los quales han hecho allianza. El Clima es mui Sano, estando la isla cerca del quinto (quincuagesimo) grado. Podría sacarse provecho de sus producciones; pero se mira como una grande ventaja, $\mathrm{q}^{\mathrm{e}}$ puedan aproar allí los Navios; $q^{\mathrm{e}}$ pretendan pasar a las grandes indias $\mathrm{p}^{\mathrm{r} .}$ la via de Norte (por la

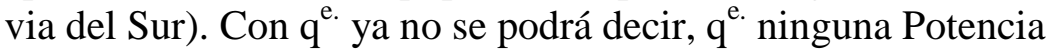

\{14\} Gaz. de lugano de 2 de Sept. 1765

[166 vta.] se ha aplicado a desfrutar las conveniencias de un Establecimiento en aquellas partes.

Y si los españoles las han tenido abandonadas desde el principio de este Siglo,

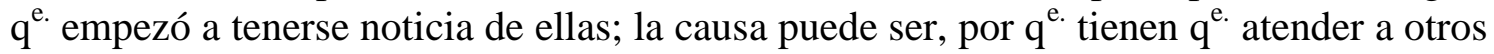
muchos Establecimientos antiguos, cuia conservación, y firmeza importa mas q empe-

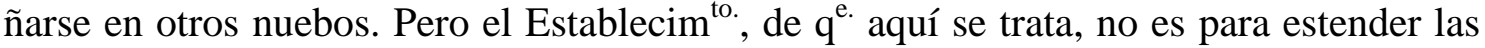
adquisiciones hechas, y ampliar la dominación, si para Redondear lo ya adquirido, defenderlo, guardarlo, y mejorarlo. 
El haver pasado tantos años sin conocerse estas tierras con caer tan cerca de los primeros descubrimientos, puede ser, por $\mathrm{q}^{\mathrm{e}}$ los Navios $\mathrm{p}^{\text {a. }}$ tomar la boca del Estrecho de Maire, havran querido asegurarse Reconociendo antes la Costa de Patagones, o alguno de los Cabos, $\mathrm{q}^{\text {e. }}$ flanquean el de Magallanes, a la manera $\mathrm{q}^{\mathrm{e} .}$ los Navios $\mathrm{q}^{\mathrm{e} .}$ vienen de indias, $\mathrm{p}^{\mathrm{a} .}$ entrar en la Bahia de Cadiz, giran para Reconocer primero el Cabo de S. Vicente. Y en aquella vuelta se ladeaban hacia el Poniente mas de lo $q^{\text {e. }}$ convenia $\mathrm{p}^{\text {a. }}$ descubrirlas. Y si esta Razon no Satisface, no será ageno Recurrir a la Providencia, $\mathrm{q}^{\mathrm{e} .}$ valiéndose de Casualidades humanas, depara tales descubrimientos quando quiere, y quando en su Eterno Consejo ha determinado abrir passo, $\mathrm{p}^{\mathrm{a} .} \mathrm{q}^{\mathrm{e} .}$ entre la luz de su conocimiento a las islas, y naciones, $\mathrm{q}^{\mathrm{e}}$ viven, o en tinieblas, o entre sombras de muerte: avisando con tales noticias a los $q^{\text {e. }}$ tienen poder, $q^{\text {e. }}$ Cooperen para dar cumplimiento a sus Misericordias.

En el hecho no hai duda; $\mathrm{q}^{\mathrm{e}}$ en las Referidas alturas, o cercanías, hai tales tierras pobladas de infieles; y q $\mathrm{q}^{\mathrm{e}}$ haviendose descubierto el Estrecho de Magallanes el año 1520; y el de Maire el de 1616., pasaron desde aquel descubrimiento 180 años, desde este 84, hasta $\mathrm{q}^{\mathrm{e}}$ las Nuevas islas se Empezaron a conocer; $\mathrm{q}^{\mathrm{e}}$ aunque algunos las han querido confundir con otras islas al parecer despobladas, e intransitables llamadas

[167] de Sebaldo Wert ${ }^{25}$ años antes, el de 1598, descubiertas, y abandonadas; pero es cierto q $\mathrm{q}^{\mathrm{e}}$ son diversas; las de Sebaldo Wert, son tres islotes, puestos en triangulo, teniendo cada uno media legua de largo, como lo Reconocio de cerca el año 1711 Un Navio de S. Maló llamado la Encarnacion: cosa $\mathrm{q}^{\mathrm{e} .}$ no tiene $\mathrm{q}^{\mathrm{e}}$. ver con los nuevos descubrimientos. De estos no tubo noticia Mr. Beauchene ${ }^{26}$ habiendo Reconocido aquellos el año de 1701 (1)

Pero dado caso, $\mathrm{q}^{\text {e. }}$ ni en lo $\mathrm{q}^{\mathrm{e} .}$ se ha descubierto delas Malvinas al Sur, al Este, y al Norte, ni en lo $\mathrm{q}^{\mathrm{e}}$ al Occidente esta $\mathrm{p}^{\mathrm{r} .}$ descubrir, se hallase Puerto y Sitio comodo para el Establecim ${ }^{\text {to. }} \mathrm{q}^{\text {e. }}$ se pretende; no $\mathrm{p}^{\text {r. }}$ eso se debería desistir del Empeño, sino ponerlo en la Tierra del Fuego, donde según las noticias, $\mathrm{q}^{\mathrm{e} .}$ dan $\operatorname{los} \mathrm{q}^{\mathrm{e}}$ naufragaron en la Reciente perdida del Navio la Concepcion, parece $\mathrm{q}^{\mathrm{e}}$ no falta ni Puerto, ni comodidad: $\mathrm{y}$ donde colocado también puede serbir a los Espresados tres fines; si bien se tiene $\mathrm{p}^{\mathrm{r} .}$ mas conveniente habiendo lugar en las Malvinas, y de estas a la parte Occidental, $\mathrm{q}^{\text {e. }}$ mira al Continente de la America. Lo mismo parece $\mathrm{q}^{\mathrm{e}}$ se debria practicar, si los Franceses ya establecidos no dejasen la tierra, como dicen $q^{\text {e. }}$ lo han prometido: y no se tuviese por conveniente partir el terreno entre las dos naciones.

En el Registro de la Costa de Patagones, $q^{\text {e. }} \mathrm{p}^{\text {r. }}$ Orden del $\mathrm{S}^{\text {or. }}$ Phelipe $\mathrm{V}^{27}$ de gloriosa memoria se hizo el año de 1745 y 46 mobio a la determinación un fin, $\mathrm{q}^{\mathrm{e}}$ toda via

\footnotetext{
${ }^{25}$ Se dice, aunque la veracidad de la afirmación sea dudosa, que el navegante neerlandés Sebald de Weert (1567-1603) habría sido quien avistó por primera vez las Malvinas en 1600, denominándolas Sebaldinas, nombre con el que las islas fueron conocidas durante un cierto tiempo (Caviglia, 2012: 60-65).

${ }^{26}$ A Jacques Gouin de Beauchêne (1652-1730) se lo recuerda por haber sido el primer navegante francés en doblar el cabo de Hornos desde el Oeste. En ocasión de hacerlo y al avanzar por aguas atlánticas hacia el nordeste descubrió además una de las pequeñas islas integrantes del archipiélago de Malvinas, que hoy lleva su nombre (Isla Beauchene), y que se halla ubicada al sur de la Isla Soledad (Caviglia, 2012: 74-75).

${ }^{27}$ Felipe duque de Anjou (1683-1746) resultó coronado rey de España a fines de 1700, y fue el primer monarca de la casa Borbón en ocupar ese trono. Durante su largo reinado se iniciaron una serie de transformaciones económicas, políticas, administrativas y científicas más tarde conocidas en conjunto bajo el nombre de reformas borbónicas, desarrolladas principalmente por su hijo y sucesor Carlos III. En el mar- 
Subsiste, y al qual con mas Razones de convivencia puede darse cumplimiento, haciendo en las Malvinas, o en la Tierra del Fugo, lo q entonces se proyecto en la Costa de Magallanes. La mira de Phelipe V (dice el P. Charlevoix (2)) en aquella Empresa era, lo primero, de saber si havia en aquella Costa Pueblos dispuestos aponerse en Reduccion para abrazar el Christianismo::::::: Lo Segundo de Examinar si havia algún puerto comodo, $\mathrm{q}^{\mathrm{e}}$ fortificado pudiese servir de Refugio alos Navios Españoles en caso de necesidad

(1) Charlev. Fast. Amn. 1700

(2) Charlev. Historia du Parag. lib. 22. pag. 173. Edic en 8 tom. 6

[167 vta.] "asegurar una Entrada fácil alo interior del Continente, e "impedir q etras naciones se estableciesen en aquellas partes. Para este fin se despacho el Orden con limitación de Registrar solamente la Costa hasta el Estrecho, no porq ${ }^{\text {e. }}$ el Establecim ${ }^{\text {to. }}$ en la Otra parte del Estrecho no pudiera servir para el mismo fin, sino $p^{\text {r. }} q^{\text {e. }}$ las noticias antecedentes, $\mathrm{q}^{\mathrm{e}}$ se tubieron presentes para aquella determinación, aseguraban, $\mathrm{q}^{\mathrm{e}}$ antes de llegar al Estrecho havia lo $\mathrm{q}^{\text {e. }}$ se buscaba. Todo se hallo falso, discordando enteramente la Realidad de los mapas, y Relaciones de los Viageros. El desengaño llego ala Corte quando aquel Monarca havia pasado a mejor vida.

En el Siguiente Reynado Ocuparon el Real animo del $S^{\text {or. }} D^{\text {n. }}$ Fernando $\mathrm{VI}^{28}$ otras providencias relativas al arreglo, y determinación de limites cerca de aquellas partes, $\mathrm{q}^{\mathrm{e}}$ duraron todo el tiempo de su vida: y no hubo tiempo para dar providencia sobre

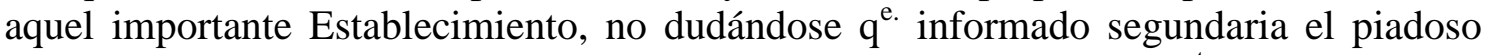
intento de su Padre, y mandaria poner en Obra o en las tierras nuebam ${ }^{\text {te. }}$ descubiertas, o en la tierra del Fuego, lo q ${ }^{\text {e. }}$ se havia visto no poder tener efecto en otra parte, y allí lo tendría mas ventajoso.

El Rey Nro $S^{\text {or. }}$ (que Dios guarde) ha heredado con el Señorio de aquellas provincias Remotas dela America la piedad, el Celo, y las Reales Virtudes de sus Augustos Predecesores, Padre y Hermano: el deseo de fomentar todo lo conducente a la Exaltacion dela fé, ala Seguridad, y Conveniencias de aquellos Estados. A sus Ministros sobran luces, $\mathrm{p}^{\mathrm{a}}$ comprehender la importancia, y Consecuencias del asunto, q $\mathrm{q}^{\mathrm{e}}$ aquí se propone. Y no se duda, $\mathrm{q}^{\mathrm{e}}$ informado S. M. de la utilidad, y tanvien de la facilidad, $\mathrm{q}^{\mathrm{e}}$ tiene la Execucion, dara Eficazes Ordenes, para $\mathrm{q}^{\mathrm{e}}$ en tan dilatado Espacio de tierras, no se diga $\mathrm{q}^{\mathrm{e}}$ no hai un Alcazar de la fé, de donde los Misioneros salgan a la Conversion de los infieles, $\mathrm{q}^{\mathrm{e}}$ andan Ciegos por aquellas partes, sin hallar quien los ponga en las Sen-

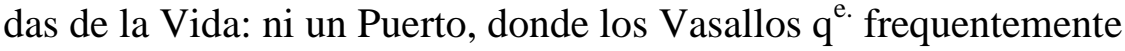

[168] Zozobran en aquellos peligrosos mares, se Recobren: ni una Fortaleza, que haga respetar de las Naciones Emular la Vandera de España.

\section{$\underline{\text { Nota }}$}

Para que la Empresa tenga el Suceso q ${ }^{\text {e. }}$ se desea, convendría, q. S. M. arreglase, y mandase observar precisam ${ }^{\text {te. }}$ las condiciones, y modo, con que se ha de Executar. Y estas condiciones parecen ser las que se ordenan en las leyes de Indias, que tratan de los

co de esas reformas, cobra sentido -tal como dijéramos- la idea de fundar una misión y puerto en las Malvinas, que fue propuesta justamente mientras gobernaba este último.

${ }^{28}$ Fernando VI (1713-1759) fue coronado sucesor de Felipe V en 1746, a raíz de los sucesivos fallecimientos de tres hermanos mayores que lo precedían en el orden dinástico. Su reinado se prolongó hasta 1759. 
Descubrimientos, y Pacificaciones, con Especialidad las Siguientes. $1^{\mathrm{a}}$ Que el Cabo, la tropa, y las personas aquien de huviere de Encargar la Execucion sean Escogidas y aprobadas en Christiandad, y deseosas de la Conversion de los indios (1). = $2^{\mathrm{a}}$ Que se lleven dos, o tres Embarcaciones, $\mathrm{q}^{\mathrm{e}}$ se pueden Engolfar, y Costear por qualesquiera Rios, y barras sin peligro de bajos; para que quedando una en el Establecim ${ }^{\text {to. }}$ pueda volver la otra a dar quenta al Gobierno (2) $=3^{\mathrm{a}}$ (3) Que el Capitan, O Cabo no proceda sin acuerdo de los Missioneros. $4^{\mathrm{a}}$ (4): Que donde bastaren los Missioneros para la pacificación, y Conversion de los indios, no se Consienta, que Entren Otras personas q ${ }^{\mathrm{e}}$ la puedan Estorbar. $5^{\mathrm{a}}$ (5): Que no se tome a los indios cosa ninguna de sus bienes, sin que primero se les pague a Satisfaccion suia. Y por que los días son mas cortos en aquella altura que en otras mas apartadas del polo, durante el Imbierno, convendria que se Ordene el viage en tiempo que pueda darse principio al Establecimiento al principio del Verano.

(1) Lib. 4. tit. 1. 1. 2. (2) Lib. 4. tit.2. 1. 2. (3) Lib. 4. tit.2. 1. 10.

(2) (4) Lib. 4. tit. 4. 1. 4. (5) Lib. 4. tit. 4. 1. 8.

\section{Referencias}

Burke, E. (1760). An account of the European settlements in America. Londres: R. \& J. Dodsley. Disponible en línea: https://babel.hathitrust.org/cgi/pt?id=aeu.ark:/13960/t10p1xm7z;view=1up;seq= 6. Última visita: 10 agosto 2015.

Byron, J. (1996) [1768]. Naufragio en las costas patagónicas. Buenos Aires: Ediciones del Sol - Ediciones UNESCO.

Caviglia, S. (2012). Malvinas: Soberanía, Memoria y Justicia. Rawson: Ministerio de Educación de la Provincia de Chubut

Charlevoix, P. F. X. de (1736). Histoire et description générale du Japon, où l'on trouvera tout ce qu'on a pu apprendre de la nature et des productions [...]Avec les Fastes chronologiques de la découverte du Nouveau Monde. París: Chez Julien Michel Gandouin. Disponible en línea: http://gallica.bnf.fr/ark:/12148/bpt6k853948v/f9.item.zoom. Última visita: 10 agosto 2015.

(1756). Histoire du Paraguay. París: Chez Didot, Giffart e Nyon. Tomos I y VI. Disponible en línea:

https://books.google.com.ar/books?id=0KwaAAAAYAAJ\&printsec=frontcover

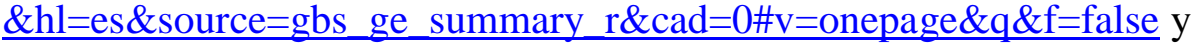
https://books.google.com.ar/books?id=oOQ-

AAAAcA-

AJ\&pg=PP7\&dq=Histoire + du + Paraguay + tome + VI\&hl=es\&sa=X\&ved=0ahUK Ewip0I--

OMPOAhVEUZAKHefQCZkQ6AEIHDAA\#v=onepage $\& \mathrm{q}=$ Histoire $\% 20 \mathrm{du} \% 20$ Paraguay\%20tome\%20VI\&f=false. Última visita: 10 agosto 2015. 
Couyoumdjian, J. R. (1971). "Manuel José de Orejuela y la abortada expedición en busca de los Cesares y extranjeros, 1780-1783”. Revista Historia, 10, pp.: 57-176.

Elkin, D. (2014). “Arqueología subacuática”. Ciencia Hoy, v. 23, n 138, pp.: 35-40.

Falkner, T. (1774). A Description of Patagonia and the adjoining parts of South Ameri$c a$, with a grammar and a short vocabulary, and some particulars relating to Falkland's Islands. Hereford: C. Pugh. Disponible en línea: https://archive.org/stream/descriptionofpat01falk\#page/n5/mode/2up. Última visita: 10 agosto 2015.

Föerster, R. (1996). Jesuitas y Mapuches: 1593-1767. Santiago de Chile: Editorial Universitaria.

Furlong SJ, G. (1984). Los jesuitas y la cultura Rioplatense. Buenos Aires: Ediciones Universidad del Salvador.

(1943). Entre los Tehuelches de la Patagonia. Buenos Aires: San Pablo.

Hanisch Espíndola SJ, W. (1972). Itinerario y pensamiento de los jesuitas expulsos de Chile, 1767-1815. Santiago de Chile: Andres Bello.

(1892), La isla de Chiloé, capitana de rutas australes. Santiago de Chile: Academia Superior de Ciencias Pedagógicas de Santiago.

Mandrini, R. (2003). "Estudio preliminar", en Falkner, 2003 [1774] Descripcion de la Patagonia y de las partes contiguas de la America del Sur, pp. 9-53.

Medina, T. (1906) Diccionario biográfico colonial del Chile. Santiago de Chile, Imprenta Elzeviriana.

Mörner, M. (1986). Actividades políticas y económicas de los Jesuitas en el Rio de la Plata. Buenos Aires: Hyspamerica.

Morris, I. (2004) [1751]. Una narración fiel de los peligros y desventuras que sobrellevó Isaac Morris. Buenos Aires: Editorial Taurus, pp. 81-129.

Nicoletti, M. A. (2014). "Pasado y presente: los jesuitas de la misión Nahuel Huapi (1670-1674 y 1704-1717) y la devoción Mariana.”. IHS. Antiguos jesuitas en Iberoamérica, vol. 2, $\mathrm{n}^{\mathrm{o}}$ 1, pp.: 41-64.

Page, C. A. (2013). "El proyecto jesuítico para la exploración y ocupación de las costas patagónicas en el Siglo XVIII”, Temas Americanistas, 30, pp.: 23-49.

Pinto Rodríguez, J. (1988). Misioneros en la Araucanía, 1600-1900: un capítulo de historia fronteriza en Chile. Temuco: Universidad de la Frontera.

(1991). Misticismo y violencia en la temprana evangelización de Chile. Temuco: Universidad de la Frontera.

Ratto, H: (1930). "Relato del naufragio del Registro "Purísima Concepción" y construcción de la goleta 'Nuestra Real Capitana San José y Las Animas' puesta en gradas en Tierra del Fuego en 1765". En: Ratto, Héctor. Actividades Marítimas en la Patagonia Austral durante los siglos XVII y XVIII. Buenos Aires: Kraft.

Santos Hernández, Á. (1992). Los Jesuitas en América. Madrid: Mapfre. 
Storni SI, H. (1980). Catálogo de los jesuitas de la provincia del Paraguay (Cuenca del Plata), 1585-1768. Roma: Institutum Historicum Societatis Iesu.

Villar, D. (2004). "Estudio preliminar. Indígenas, españoles e ingleses en el Río de la Plata y Chile durante el siglo XVIII. Acerca de la Narración fiel de los peligros y desventuras que sobrellevó Isaac Morris: textos y contextos”, en Morris, 2004 [1751], pp. 9-68.

Vosgien \& Echard (1749). Dictionnaire Géographique Portatif. París: Chez Didot. Disponible en línea: https://books.google.com.ar/books?id=2Y28wJEJgIC\&printsec $=$ frontcover\&hl $=$ es\&source $=$ gbs_ge_summary_r\&cad $=$ $\underline{0 \# \mathrm{v}=\text { onepage } \& \mathrm{q} \& \mathrm{f}=\text { false. }}$. Última visita: 10 agosto 2015.

(1757). Dizionario geográfico portatile. Venecia: nell stamperia Remondini. Disponible en línea: https://books.google.com.ar/books?id=860p48CIz4UC\&printsec=frontcover\&hl $=e s \&$ source $=g b s \_g e \_s u m m a r y \_r \& c a d=0 \# v=$ onepage $\& q \& f=f a l s e$. Última visita: 10 agosto 2015 .

Walter, R. (1748). A voyage round the World, in the Years MDCCXL, I, II, III, IV, by George Anson, Esq.; Now Lord Anson, Commander in Chief of a Squadron of His Majesty's Ships, sent upon an Expedition to the South-Seas. Compiled From his Papers and Materials, by Richard Walter, M. A. Chaplain of His Majesty's Ship the Centurion, in that Expedition. Ilustred with Forty Two Copper-Plates. London: Printed for the Author By John and Paul Krapton, in Ludgate-Street.

Wright, J. (2005). Los jesuitas: una historia de los "soldados de Dios". Buenos Aires: Debate. 